\title{
FGF-17 from Hypoxic Human Wharton's Jelly-Derived Mesenchymal Stem Cells Is Responsible for Maintenance of Cell Proliferation at Late Passages
}

\author{
Kyu-Hyun Han ${ }^{1}$, Min-Hee Kim ${ }^{1}$, Gun-Jae Jeong ${ }^{1}$, Ae-Kyeong Kim ${ }^{1}$, Jong Wook Chang ${ }^{2}$, Dong-ik Kim ${ }^{1}$ \\ ${ }^{I}$ Division of Vascular Surgery, Samsung Medical Center, Sungkyunkwan University School of Medicine, Seoul, Korea \\ ${ }^{2}$ Stem Cell \& Regenerative Medicine Institute, Research Institute for Future Medicine, Samsung Medical Center, Seoul, Korea
}

Background and Objectives: Although it is well known that hypoxic culture conditions enhance proliferation of human mesenchymal stem cells, the exact mechanism is not fully understood. In this study, we investigated the effect of fibroblast growth factor (FGF)-17 from hypoxic human Wharton's Jelly-derived mesenchymal stem cells (hWJ-MSCs) on cell proliferation at late passages.

Methods and Results: hWJ-MSCs were cultured in $\alpha$-MEM medium supplemented with 10\% fetal bovine serum (FBS) in normoxic $\left(21 \% \mathrm{O}_{2}\right)$ and hypoxic $\left(1 \% \mathrm{O}_{2}\right)$ conditions. Protein antibody array was performed to analyze secretory proteins in conditioned medium from normoxic and hypoxic hWJ-MSCs at passage 10. Cell proliferation of hypoxic hWJ-MSCs was increased compared with normoxic hWJ-MSCs from passage 7 to 10, and expression of secretory FGF-17 was highly increased in conditioned medium from hypoxic hWJ-MSCs at passage 10. Knockdown of FGF-17 in hypoxic and normoxic hWJ-MSCs decreased cell proliferation, whereas treatment of hypoxic and normoxic hWJ-MSCs with recombinant protein FGF-17 increased their proliferation. Signal transduction of FGF-17 in hypoxic and normoxic hWJ-MSCs involved the ERK1/2 pathway. Cell phenotypes were not changed under either condition. Differentiation-related genes adiponectin, Runx2, and chondroadherin were downregulated in normoxic hWJ-MSCs treated with rFGF-17, and upregulated by siFGF-17. Expression of alkaline phosphatase (ALP), Runx2, and chondroadherin was upregulated in hypoxic hWJ-MSCs, and this effect was rescued by transfection with siFGF-17. Only chondroadherin was upregulated in hypoxic hWJ-MSCs with rFGF-17.

Conclusions: In hypoxic culture conditions, FGF-17 from hypoxic hWJ-MSCs contributes to the maintenance of high proliferation at late passages through the $\mathrm{ERK1} / 2$ pathway.

Keywords: FGF-17, Hypoxic culture, Mesenchymal stem cells, Proliferation

Received: May 12, 2018, Revised: February 27, 2019,

Accepted: March 4, 2019, Published online: April 30, 2019

Correspondence to Dong-ik Kim

Division of Vascular Surgery, Samsung Medical Center, Sungkyunkwan University School of Medicine, 81 Irwon-ro, Gangnam-gu, Seoul 06351, Korea

Tel: +82-2-3410-3467, Fax: +82-2-3410-0040

E-mail: dikim@skku.edu

(a) This is an open-access article distributed under the terms of the Creative Commons Attribution Non-Commercial License (http://creativecommons.org/ licenses/by-nc/4.0/), which permits unrestricted non-commercial use, distribution, and reproduction in any medium, provided the original work is properly cited.

Copyright (C) 2019 by the Korean Society for Stem Cell Research

\section{Introduction}

Hypoxic culture conditions enhance the proliferation or survival of several cell types such as tumor cells $(1,2)$, endothelial cells (3), fibroblasts (4), neural stem cells (5), and mesenchymal stem cells (6). Moreover, cellular proliferation is maintained at a high level in long-term hypoxic culture (7).

Although a signal pathway dependent on hypoxia-inducible factor (HIF)-1 $\alpha$ or HIF-2 $\alpha$ has been reported as the major mechanism for high proliferation in hypoxic 
conditions $(8,9)$, identification of other involved signal pathways or molecules is required to understand the response and function of cells under hypoxic condition. The secretome from mesenchymal stem cells in hypoxic culture condition shows beneficial effects on the cells themselves or neighboring cells through autocrine or paracrine signaling (10-12).

Previous studies have reported that fibroblast growth factor (FGF)-17 is expressed in the embryonic brain (13). Moreover, FGF17 increased the proliferation of carcinoma cells (14) and leukemic cells (15), and inhibited the differentiation of oligodendrocyte progenitor cells (16). However, the role of FGF-17 in human mesenchymal stem cells cultured in hypoxic conditions has not yet been investigated.

In this study, we aimed to investigate the role of FGF-17 secreted by human Wharton's Jelly-derived mesenchymal stem cells (hWJ-MSCs) cultured in hypoxic conditions at late passages based on protein profiling of conditioned medium (CM) of hypoxic hWJ-MSCs.

\section{Materials and Methods}

\section{Cell cultures}

This study was approved by the Institutional Review Board of Samsung Medical Center and informed consent was obtained from pregnant mothers (IRB. No.201607-102). hWJ-MSCs were isolated according to the procedure specified in a previous report (17) and cultured in Alpha Minimum Essential Medium ( $\alpha$-MEM, Gibco, Carlsbad, CA, USA) supplemented with 10\% FBS (Gibco) and $0.5 \%$ antibiotic/antimycotic (Gibco) at $37^{\circ} \mathrm{C}$ in normoxic $\left(21 \% \mathrm{O}_{2}\right)$ or hypoxic $\left(1 \% \mathrm{O}_{2}\right)$ culture conditions with $5 \% \mathrm{CO}_{2}$. Media changes and passaging for both conditions were performed in a normoxic environment. When the culture reached $90 \%$ confluency, the cells were washed twice with phosphate buffered saline (PBS) and dissociated with $0.25 \%$ trypsin-EDTA (Gibco) for 2 minutes at $37^{\circ} \mathrm{C}$. Dissociated cells were treated with medium to block trypsin-EDTA activity. After centrifugation, cell pellets were resuspended with medium and seeded at 3,000 cells $/ \mathrm{cm}^{2}$. Cell images were acquired with a model CKX41 inverted microscope (Olympus Corporation, Tokyo, Japan). For the supplementary experiments on the role of FGF-17 in hWJ-MSCs, 2 of hWJ-MSCs were purchased from PromoCell (Heidelberg, Germany) and named as hWJMSCs-1 and hWJ-MSCs-2.

\section{Protein antibody array of CM from hWJ-MSCs}

Normoxic or hypoxic hWJ-MSCs at passage 10 were seeded in $100-\mathrm{mm}$ diameter culture plates containing com- plete medium at a density of 3,000 cells $/ \mathrm{cm}^{2}$. When the cells reached $90 \%$ confluency the medium was replaced with $12 \mathrm{ml}$ of serum-free medium. After incubation for 18 $\mathrm{h}, \mathrm{CM}$ was concentrated by centrifugation at $5,000 \times \mathrm{g}$ for $1 \mathrm{~h}$ using an Amicon Ultra-15 centrifugal filter (Millipore, Billerica, MA, USA) with a size cut-off of 3,000 Dalton. Expression of proteins in CM from hWJ-MSCs was measured with a RayBio ${ }^{\circledR}$ Label-based (L-Series) Human Antibody Array (Raybiotech, Inc., Norcross, GA, USA) from E-biogen (Kyung Hee Business Center, Kyung Hee University, Seoul, Korea). The result was acquired from a single sample analysis. Upregulated and downregulated proteins are listed in Fig. 1B, 1C and Table 1.

\section{ELISA}

Expression of FGF-17 in CM from normoxic hWJ-MSCs or hypoxic hWJ-MSCs at passage 10 was measured using Human FGF-17 ELISA Kit, Cusabio Technology, LLC, College Park, MD, USA) by manufacture's manual. Briefly, 100 ul of $1: 3$ diluted CM from normoxic or hypoxic $\mathrm{hWJ}$-MSCs at passage 10 were added to each well of 96 well plate coated with anti-FGF-17 antibody, and incubated for $2 \mathrm{~h}$ at $37^{\circ} \mathrm{C}$. In the next step, biotin-antibodies were added to each well for $1 \mathrm{~h}$ at $37^{\circ} \mathrm{C}$. After washing wells with washing buffer, HRP-avidins were added to each well for $1 \mathrm{~h}$ at $37^{\circ} \mathrm{C}$. TMB substrates were added to each well for $15 \mathrm{~min}$ and stop solutions were added. The optical density of each well was determined using a microplate reader xMark microplate spectrophotometer (BioRad, Hercules, CA, USA) set to $450 \mathrm{~nm}$.

\section{Treatment with siFGF-17 and rFGF-17}

For transfection with siRNA, 20 pmol of siFGF-17 (sense, CGACUGAAGGCCUUGCAGA; antisense, UCUG CAAGGCCUUCAGUCG; Bioneer, Daejeon, Korea) was mixed with 1 ul of Lipofectamin 2000 (Invitrogen, Carlsbad, CA, USA) for 20 minutes at room temperature, and then added to normoxic hWJ-MSCs at passage 7 and hypoxic $\mathrm{hWJ}$-MSCs at passage 10. After $6 \mathrm{~h}$, the medium was replaced with fresh complete medium, and the cells were cultured for $48 \mathrm{~h}$. Negative control siRNA was used for the control group (SN-1013, Bioneer, Daejeon, Korea).

For rFGF-17 treatment, normoxic hWJ-MSCs at passage 7 and hypoxic hWJ-MSCs at passage 10 were treated with 250, 500, 1,000 ng/ml of rFGF-17 (PeproTech Inc., Rocky Hill, NJ, USA) for $48 \mathrm{~h}$.

For both treatments, cell counting, BrdU proliferation assay, quantitative real-time polymerase chain reaction (QRT-PCR), western blot, and flow cytometry analysis were performed after incubation for $48 \mathrm{~h}$, as described 
A

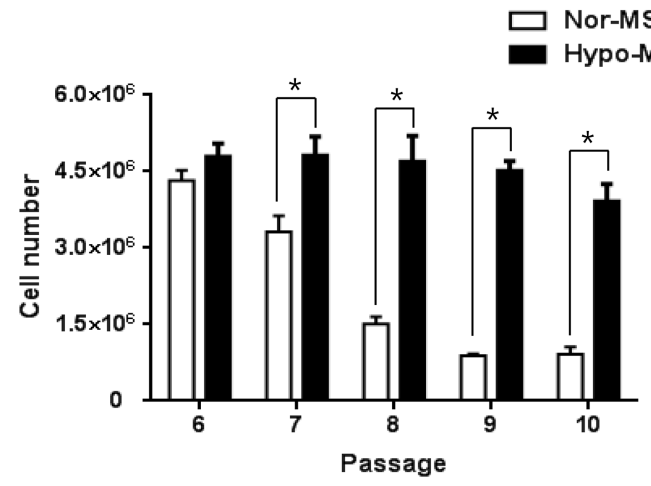

C

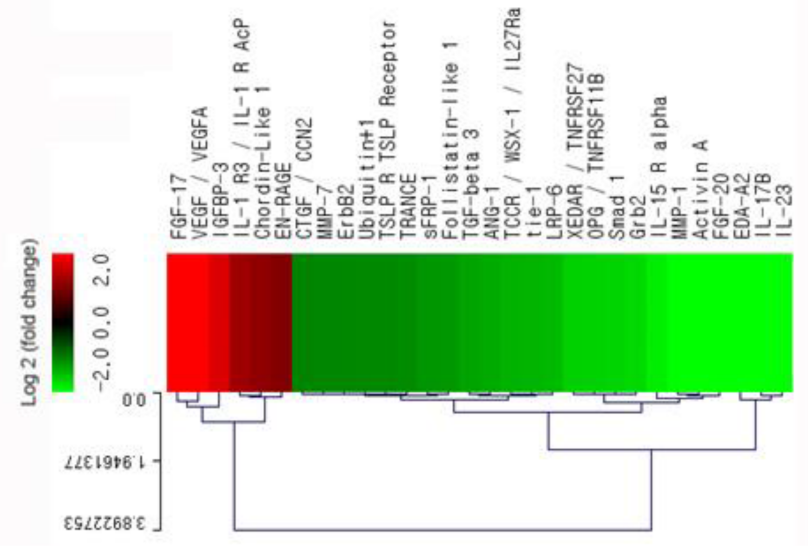

D

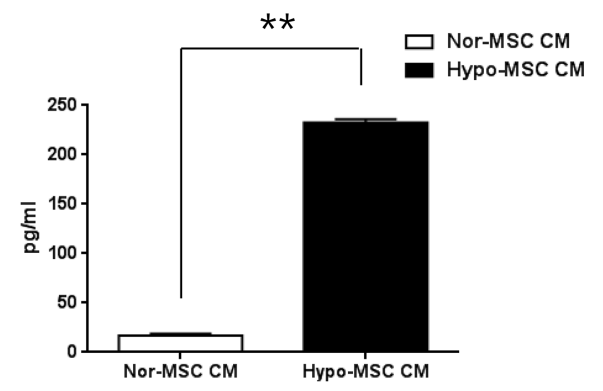

below.

\section{Cell proliferation assay}

Cells from passages 6 to 10 were seeded at 3,000 cells $/ \mathrm{cm}^{2}$ in normoxic or hypoxic culture conditions. Cells were removed by trypsinization at $90 \%$ confluency for each passage and cell numbers were counted using Trypan blue $0.5 \%$ solution (Biowest, Riverside, MO, USA) and a hemocytometer. A BrdU cell proliferation assay kit (Millipore) was used to analyze cell proliferation according to the manufacturer's manuals. Briefly, normoxic or hypoxic $\mathrm{hWJ}$-MSCs were seeded in 96-well plates at 3,000 cells $/ \mathrm{cm}^{2}$, USA).
B

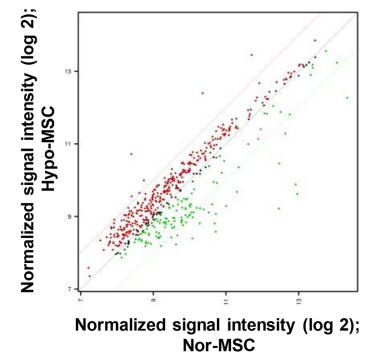

Fig. 1. Enhanced proliferation of hypoxic hWJ-MSCs and levels of secretory proteins at late passages. (A) Cell numbers of normoxic and hypoxic hWJ-MSCs from passage 6 to 10. Results are expressed as the mean \pm SEM from three independent experiments $\left(\mathrm{n}=3,{ }^{*} \mathrm{p}<0.05\right.$, t-test). (B) Scatterplot of protein expression levels between normoxic and hypoxic $h W J-M S C s$ at passage 10 . The red line above represents upregulated proteins ( $>2$ fold), and the green line below represents downregulated proteins ( $>2$ fold) in conditioned medium from hypoxic hWJMSCs compared with normoxic hWJMSCs. (C) Clustering of upregulated proteins and downregulated proteins in conditioned medium from hypoxic hWJ-MSCs compared with normoxic hW)-MSCs at passage 10 (fold change $>2.0$ ). Results were from a single sample analysis. (D) Absolute amount of FGF-17 in CM from normoxic hWJ-MSCs and hypoxic hWJ-MSCs $(1: 3$ diluted CM) $(\mathrm{n}=3, * * \mathrm{p}<0.01$, t-test). (E) Expression of FGFR-1, -2, -3 and -4 in normoxic and hypoxic hWJ-MSCs at passage 10. Nor-MSC, normoxic mesenchymal stem cells; Hypo-MSC, hypoxic mesenchymal stem cells.

incubated overnight, and treated with $1 \times$ BrdU solution for $24 \mathrm{~h}$. The medium was removed and the cells were treated with Fixing/Denaturing Solution (Millipore) for $30 \mathrm{~min}$. Cells were incubated with $1 \times$ detection antibody and $1 \times$ HRP-conjugated secondary antibody (Millipore) for $1.5 \mathrm{~h}$ and washed with $1 \times$ Wash Buffer (Millipore) After incubation with TMB Substrate (Millipore) for 30 min, STOP solution (Millipore) was added and the absorbance of BrdU was measured at $450 \mathrm{~nm}$ using xMark Microplate Spectrophotometer (Bio-Rad, Hercules, CA, 
Table 1. Secretory proteins that were upregulated or downregulated ( $>2$-fold) in conditioned medium from hypoxic hWJMSCs compared with normoxic hWJ-MSCs

\begin{tabular}{lcl}
\hline \multicolumn{1}{c}{ Name } & Fold change & SwissProt ID \\
\hline FGF-17 & +5.008 & O60258 \\
VEGF/NEGFA & +4.117 & P15692 \\
IGFBP-3 & +3.340 & P17936 \\
IL-1 R3/IL-1 R AcP & +2.382 & Q9NPH3 \\
Chordin-like 1 & +2.220 & Q9BU40 \\
EN-RAGE & +2.051 & P80511 \\
CTGF/CCN2 & -2.039 & P29279 \\
MMP-7 & -2.082 & P09237 \\
ErbB2 & -2.093 & P04626 \\
Ubiquitin+1 & -2.098 & P0CG47 \\
TSLP R, TSLP Receptor & -2.107 & Q9HC73 \\
TRANCE & -2.151 & O14788 \\
sFRP-1 & -2.272 & Q8N474 \\
Follistatin-like 1 & -2.289 & Q12841 \\
TGF-beta 3 & -2.437 & P10600 \\
ANG-1 & -2.538 & Q15389 \\
TCCR/WSX-1/IL27Ra & -2.634 & Q6UWB1 \\
Tie-1 & -2.662 & P35590 \\
LRP-6 & -2.760 & O75581 \\
XEDAR/TNFRSF27 & -3.084 & Q9HAV5 \\
OPG/TNFRSF11B & -3.142 & O00300 \\
Smad 1 & -3.200 & Q15797 \\
Grb2 & -3.286 & P62993 \\
IL-15 R alpha & -3.676 & Q13261 \\
MMP-1 & -4.022 & P03956 \\
Activin A & -4.197 & P08476 \\
FGF-20 & -4.402 & Q9NP95 \\
EDA-A2 & -8.219 & Q92838 \\
IL-17B & -9.443 & Q9UHF5 \\
IL-23 & -10.144 & Q9NPF7 \\
\hline
\end{tabular}

\section{Cell viability assay}

3,000 cells $/ \mathrm{cm}^{2}$ of normoxic hWJ-MSCs at passage 7 or hypoxic $\mathrm{hWJ}-\mathrm{MSC}$ at passage 10 were seeded into the well of 96-well white plates. After $48 \mathrm{~h}$, culture medium was removed and serum free medium was added to the wells. After 1 day incubation, $500 \mathrm{ng} / \mathrm{ml}$ of $\mathrm{rFGF}-17$ was treated to the cells for $48 \mathrm{~h}$. For the inhibition of secretory FGF-17 from hypoxic hWJ-MSCs at passage 10, 6,000 cells $/ \mathrm{cm}^{2}$ of hypoxic $\mathrm{hWJ}-\mathrm{MSCs}$ were incubated in 96-well white plates for $48 \mathrm{~h}$. Then, $30 \mathrm{ug} / \mathrm{ml}$ of blocking antibody of FGF-17 (R\&D Systems, Minneapolis, MN, USA) in serum free medium was treated to the cells for $48 \mathrm{~h}$. In the next step, $100 \mathrm{ul}$ of CellTiter-Glo assay 2.0 reagents (Promega, Madison, WI, USA) was treated to the cells. After $10 \mathrm{~min}$ incubation, luminescence from cells was measured with GLOMAX Multi Detection System (Promega, Sunnyvale, CA, USA).
Table 2. Primer sequences used for QRT-PCR

\begin{tabular}{ll}
\hline \multicolumn{1}{c}{ Primer name } & \multicolumn{1}{c}{ Sequence $\left(5^{\prime} \rightarrow 3^{\prime}\right)$} \\
\hline PCNA Forward & TCCTGTGCAAAAGACGGAGT \\
PCNA Reverse & CATCCTCGATCTTGGGAGCC \\
Ki67 Forward & CAGATGATGGAGCCCGGAA \\
Ki67 Reverse & TGCACACCTCTTGACACTTCC \\
Adiponectin Forward & AACATGCCCATTCGCTTTACC \\
Adiponectin Reverse & TAGGCAAAGTAGTACAGCCCA \\
ALP Forward & TACAAGGTGGTGGGCGGTGAACGA \\
ALP Reverse & TGGCGCAGGGGCACAGCAGAC \\
Runx2 Forward & TCTCACAAATCCTCCCC \\
Runx2 Reverse & TGGATTAAAAGGACTTGG \\
Chondroadherin Forward & GGACCACAACAAGGTCACTGA \\
Chondroadherin Reverse & GTGGAATTGGCGAGGTTCTC \\
SOX9 Forward & GAACGCACATCAAGACGGAG \\
SOX9 Reverse & TCTCGTTGATTCGCTGCTC \\
$\beta$-actin Forward & AGTCCTGTGGCATCCACGAA \\
$\beta$-actin Reverse & GATCCACACGGAGTACTTGC \\
\hline
\end{tabular}

\section{Quantitative real-time polymerase chain reaction (QRT-PCR)}

Total RNA was extracted from cells using a RNeasy Plus Mini Kit (Qiagen GmbH, Hilden, Germany) and 200 ng of total RNA was used for reverse transcription with a PrimeScript RT reagent kit (Takara, Bio, Shiga, Japan) according to the manufacturer's manuals. Real- time PCR was conducted using SYBR Green Realtime PCR Master Mix (Toyobo Co., LTD, Osaka, Japan) with 100 ng template DNA in a 7900 HT Fast Real-time PCR system (Applied Biosystems, Foster City, CA, USA) using reaction conditions of denaturation at $95^{\circ} \mathrm{C}$ for $15 \mathrm{~s}$ and extension at $60^{\circ} \mathrm{C}$ for $34 \mathrm{~s}$ for 40 cycles. The primer sequences are listed in Table 2.

\section{Western blotting}

For the analysis of FGF-17 receptors on normoxic hWJMSCs and hypoxic hWJ-MSCs at passage 10, cell lysates were harvested from both kinds of cells. For the analysis of intracellular signaling related with FGF-17, cell lysates were harvested from normoxic hWJ-MSCs treated with rFGF-17 and transfected with siRNA against FGF-17 at passage 7 or hypoxic hWJ-MSCs treated with rFGF-17 and transfected with siRNA against FGF-17 at passage 10 using lysis buffer $(20 \mathrm{mM}$ HEPES $\mathrm{pH}$ 7.6, 20\% Glycerol, $250 \mathrm{mM} \mathrm{NaCl}, 1.5 \mathrm{mM} \mathrm{MgCl} 2,0.1 \%$ Triton X-100, $2 \mathrm{mM}$ PMSF, $1 \mathrm{mM}$ DTT, $1 \mathrm{mM} \mathrm{NaF}$ and $1 \mathrm{mM} \mathrm{Na}_{3} \mathrm{VO}_{4}$ ) with protease inhibitor cocktail (Roche Diagnostics $\mathrm{GmbH}$, Mannheim, Germany). Quantification of proteins in lysates was performed with Quick Start Bradford $1 \times$ Dye Reagent (Bio-Rad), and absorbance was measured at 450 
nm using xMark Microplate Spectrophotometer. Protein samples were boiled at $95^{\circ} \mathrm{C}$ for $15 \mathrm{~min}$ and $20 \mathrm{ug}$ of protein from each sample was subjected to SDS-PAGE. Separated proteins in the gel were transferred to a nitrocellulose membrane, which was incubated for $1 \mathrm{~h}$ with $5 \%$ bovine serum albumin (Abcam, Cambridge, UK) in $1 \times \mathrm{TBS}$ solution (Intron Biotechnology, Seoul, Korea) with 0.1\% Tween 20 (Sigma-Aldrich, St Louis, MO, USA). The membrane was washed with $1 \times$ TBST and incubated overnight at $4^{\circ} \mathrm{C}$ with the following primary antibodies: anti-FGFR-1, FGFR-2, FGFR-3 and FGFR-4 (1 : 1,000; Cusabio Technology, LLC, College Park, MD, USA), anti-phospho AKT (S473) (1 : 2,000; Cell Signaling Technology, MA, USA), anti-phospho ERK1/2 (Thr202/Tyr204) (1: 500; Santa Cruz Biotechnology, Santa Cruz, CA, USA), anti- ERK1/2 (1 : 1000; Abcam, Cambridge, UK), anti-phospho STAT3 (Y705) (1 : 1,000; Cell Signaling Technology), anti-GAPDH ( 1 : 10,000; Abcam, Cambridge, UK), anti-P21 (1 : 1,000; Cell Signaling Technology), anti-P27 rabbit antibody (1 : 1,000; Cell Signaling Technology), anti-P53 (1 : 500; Santa Cruz Biotechnology), and anti-FGF-17 mouse (1 : 500; Santa Cruz Biotechnology) antibody. After incubation with goat anti-mouse or -rabbit HRP-conjugated antibody (1 : 10,000; Bethyl, Montgomery, TX, USA) for $1 \mathrm{~h}$ at room temperature, the expression of proteins was visualized using WESTSAVE UP (Abfrontier, Seoul, Korea) and developed with Automatic X-RAY Film Processor (JPI Healthcare Co, Ltd., Seoul, Korea).

\section{Flow cytometry}

Normoxic hWJ-MSCs treated with rFGF-17 and transfected with siFGF-17 at passage 7 or hypoxic hWJ-MSCs treated with $\mathrm{rFGF}-17$ and transfected with siFGF-17 at passage 10 were harvested and washed with $1 \times \mathrm{PBS}$ (Intron Biotechnology, Seoul, Korea). Normoxic hWJ-MSCs not treated with $\mathrm{rFGF}-17$ and transfected with negative control siRNA or hypoxic hWJ-MSCs not treated with rFGF-17 and transfected with negative control siRNA were used as respective control groups. Cells were fixed with BD Cytofix Fixation Buffer (BD Biosciences, Piscataway, NJ, USA) and stained with V450 mouse anti-human CD31 (1:20), fluorescein isothiocyanate (FITC) mouse anti-human CD34 (1:20), phycoerythrin (PE)-Cy ${ }^{\mathrm{TM}} 7$ mouse anti-human CD44 (1 : 20), V500 mouse anti-human CD45 (1:20), PerCP-Cy ${ }^{\mathrm{TM}} 5.5$ mouse anti-human CD73 (1 : 20), PE mouse anti-human CD90 (1 : 20), APC mouse anti-human CD105 (1: 20), and V450 mouse anti-human CD144 (1:20) (BD Biosciences) antibodies for $30 \mathrm{~min}$ at room temperature. Cells were washed twice with Stain Buffer (BD Biosciences) and analyzed with a
FACSVerse $^{\mathrm{TM}}$ flow cytometer (BD Biosciences) and Flowjo software (Treestar, San Carlos, CA, USA).

\section{Statistical analysis}

Data analysis and statistics (t-tests) were conducted with GraphPad Prism version 6.01 (San Diego, CA, USA) and a p-value $<0.05$ was considered statistically significant.

\section{Results}

hWJ-MSCs cultured in hypoxic condition showed high proliferation and secretion of FGF-17 at late passages

In this study, we defined passage 6 as early passage, passages 7 and 8 as middle passages, and passages 9 and 10 as late passages for normoxic and hypoxic hWJ-MSCs. When normoxic and hypoxic hWJ-MSCs were cultured from passage 6 to 10, cell numbers of hypoxic hWJ-MSCs were highly maintained from passage 7 to 10 compared with normoxic hWJ-MSCs (Fig. 1A). To investigate the factors related to maintenance of high proliferation of hypoxic hWJ-MSCs, we compared proteins in CM from hypoxic $\mathrm{hWJ}-\mathrm{MSC}$ and normoxic hWJ-MSCs at passage 10 using protein antibody array. The signal intensities of proteins that were upregulated or downregulated by at least 2-fold were analyzed in a scatter plot (Fig. 1B). FGF-17 $(+5.008$ fold $)$, VEGF/VEGFA $(+4.117$ fold $)$, IGFBP3 $(+$ 3.340 fold), IL-1 R3/IL-1 R AcP (+2.382 fold), ChordinLike $1(+2.220$ fold), and EN-RAGE $(+2.051$ fold) were highly expressed in CM of hypoxic hWJ-MSCs (Fig. 1C, Table 1). We focused on the highest expression of FGF-17 in CM from hypoxic hWJ-MSCs, and confirmed that the absolute amount of FGF-17 in CM from hypoxic hWJMSCs was significantly higher than in CM from normoxic hWJ-MSCs (Fig. 1D). Furthermore, expression of FGF-17 in hypoxic hWJ-MSCs-1 and -2 was higher than normoxic hWJ-MSCs-1 and -2 (Supplemental Fig. S1A, S2A). Expression of the receptors of FGF-17 such as FGFR-1, -2, -3 and -4 was confirmed without different expression level in both kinds of cells (Fig. 1E).

\section{FGF-17 of hypoxic hWJ-MSCs had a role in the increase of cell proliferation}

Based on results showing the highest expression of FGF-17 in CM from hypoxic hWJ-MSCs and maintenance of high proliferation of hypoxic hWJ-MSCs at passage 10, we investigated the relationship between FGF-17 and proliferation of hypoxic hWJ-MSCs using siRNA knockdown. Expression of FGF-17 was efficiently decreased when FGF-17 was knocked down in hypoxic hWJ-MSCs by siRNA treatment (Fig. 2A). Furthermore, 
A

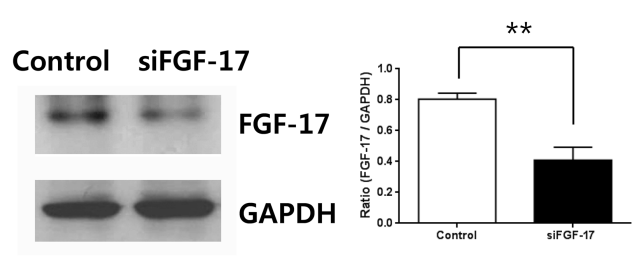

B

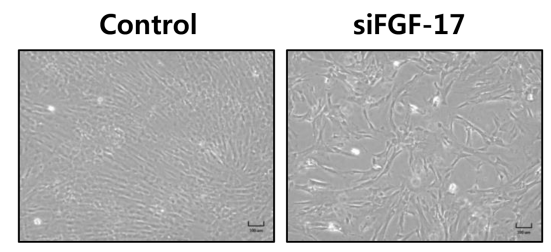

C

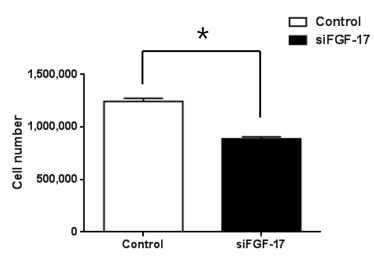

D

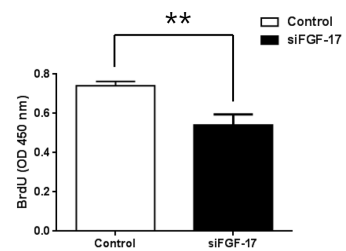

$\mathbf{F}$
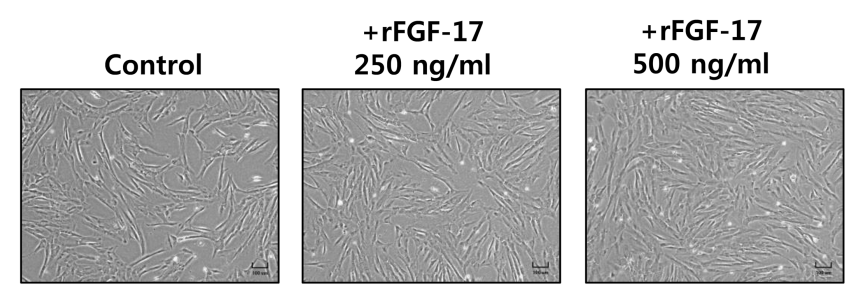

G

H

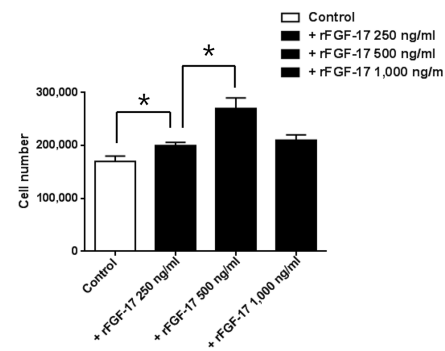

cell numbers (Fig. 2B, 2C), BrdU incorporation (Fig. 2D), and expression of proliferation-related gene Ki67 (Fig. 2E) were significantly reduced in hypoxic hWJ-MSCs transfected with siRNA of FGF-17. When hypoxic hWJ-MSCs were treated with $\mathrm{rFGF}-17$ for $48 \mathrm{~h}$, cell proliferation (Fig. $2 \mathrm{~F}, 2 \mathrm{G}$ ) and cell viability (Fig. $2 \mathrm{H}$ ) were increased in a dose-dependent manner up to $500 \mathrm{ng} / \mathrm{ml}$. Furthermore, there were consistent results on the role of FGF-17 in hypoxic hWJ-MSCs-1 and -2 (Supplementary Fig. S1B $\sim$ G, $\mathrm{S} 2 \mathrm{~B} \sim \mathrm{G})$. When secretory FGF-17 from hypoxic hWJ-MSCs was inhibited by blocking antibody of FGF-17, cell viability was significantly decreased (Fig. 2I).

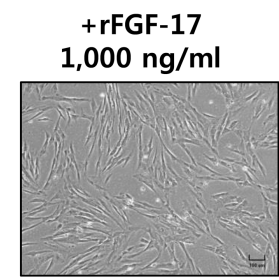

E

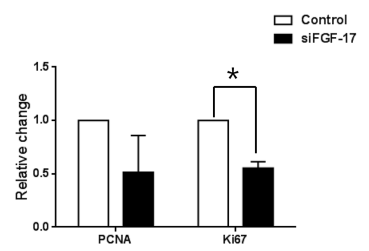

Fig. 2. FGF-17 of hypoxic hWJ-MSCs had a role in the increase of cell proliferation. Hypoxic hWJ-MSCs were transfected with siFGF-17 at passage 10 for $48 \mathrm{~h}$. (A) Expression of FGF-17. (B) Images of cells. (C) Cell number and (D) BrdU staining. (E) PCNA and Ki67 expression. Control, negative control siRNA; siFGF-17, siRNA targeting FGF-17. Hypoxic hWJ-MSCs were treated with rFGF-17 at passage 10 for $48 \mathrm{~h}$, (F) Images of cells. (G) Cell number and $(\mathrm{H})$ Cell viability $(500 \mathrm{ng} / \mathrm{ml}$ of $\mathrm{rFGF}-17$ treatment). (I) Cell viability of hypoxic hWJ-MSCs with blocking antibody of FGF-17. Control, untreated hypoxic hWJ-MSCs; + rFGF-17, hypoxic hWJMSCs treated with rFGF-17; +FGF$17 \mathrm{Ab}$, hypoxic $\mathrm{hWJ}$-MSCs with blocking antibody of FGF-17. Results are expressed as the mean \pm SEM from three independent experiments $(\mathrm{n}=3$, ${ }^{*} \mathrm{p}<0.05,{ }^{* *} \mathrm{p}<0.01$, t-test).

\section{FGF-17 of normoxic hWJ-MSCs had a role in the increase of cell proliferation}

Based on the high levels of FGF-17 in conditioned medium from hypoxic hWJ-MSCs, we investigated the effects of FGF-17 on normoxic hWJ-MSCs at passage 7. Expression of FGF-17 was efficiently decreased when FGF-17 was knocked down in normoxic hWJ-MSCs (Fig. 3A). Cell numbers (Fig. 3B, 3C) and BrdU incorporation (Fig. 3D) of normoxic hWJ-MSCs with transfected with siRNA of FGF-17 were significantly reduced. Treatment of normoxic hWJ-MSCs with rFGF-17 for $48 \mathrm{~h}$ increased cell proliferation in a dose-dependent manner up to $500 \mathrm{ng} / \mathrm{ml}$ (Fig. 3E, 3F). At 1,000 ng/ml rFGF-17, the proliferation of normoxic hWJ-MSCs was decreased, but without sig- 
A

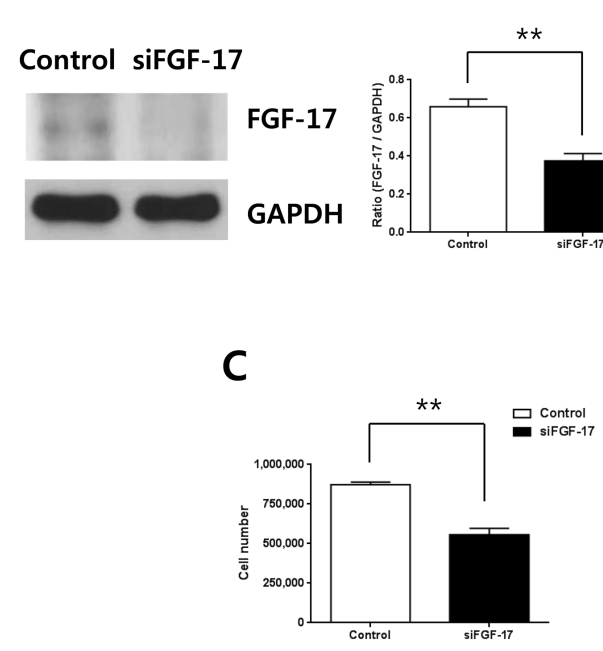

E
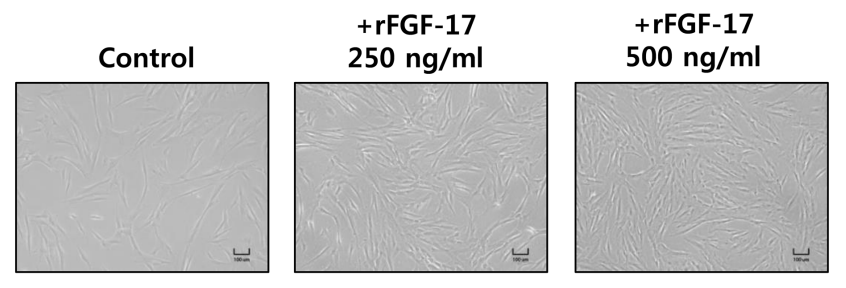

+ rFGF-17 $1,000 \mathrm{ng} / \mathrm{ml}$

$\mathbf{F}$

G
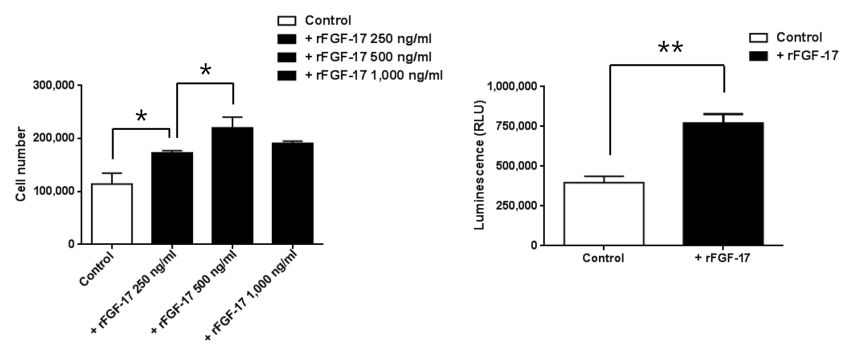

nificance (Fig. 3E, 3F). Cell viability and expression of $P C N A$ and Ki67 increased after treatment with $500 \mathrm{ng} / \mathrm{ml}$ rFGF-17 (Fig. 3G, 3H). Furthermore, there were consistent results on the role of FGF-17 in normoxic hWJ-MSCs-1 and -2 (Supplementary Fig. S1H $\sim$ M, S2H $\sim M$ ).

\section{FGF-17 affects the ERK signaling pathway in hypoxic and normoxic hWJ-MSCs}

To investigate signal transduction pathways of FGF-17 in normoxic or hypoxic hWJ-MSCs, we analyzed the expression of the following proteins related to cell proliferation or survival: phospho-AKT (S473), phospho-ERK1/2 (Thr202/Tyr204), and phospho-STAT3 (Y705). Knockdown
H

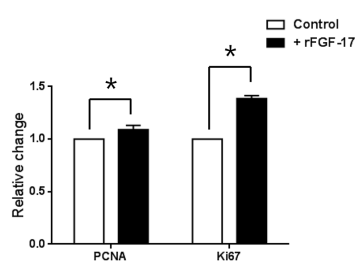

Fig. 3. FGF-17 of normoxic hWJMSCs had a role in the increase of cell proliferation. Normoxic hWJ-MSCs were transfected with siFGF-17 at passage 7 for $48 \mathrm{~h}$. (A) Expression of FGF-17. (B) Images of cells. (C) Cell number and (D) BrdU staining. Control, negative control siRNA; siFGF17, siRNA targeting FGF-17. Normoxic hWJ-MSCs were treated with rFGF-17 at passage 7 for $48 \mathrm{~h}$. (E) Images of cells. (F) Cell numbers. (G) Cell viability $(500 \mathrm{ng} / \mathrm{ml}$ of rFGF-17 treatment). (H) PCNA and Ki67 expression. Control, untreated normoxic hWJ-MSCs; +rFGF-17, normoxic hWJMSCs treated with rFGF-17. Results are expressed as the mean \pm SEM from three independent experiments $(\mathrm{n}=3$, ${ }^{*} \mathrm{p}<0.05, *{ }^{*} \mathrm{p}<0.01$, t-test). of FGF-17 in hypoxic (Fig. 4A, 4B) or nomoxic hWJ-MSCs (Fig. 4E, 4F) decreased expression of phospho-ERK1/2 (Thr202/Tyr204), but had no effect on levels of phospho-AKT (S473) and phospho-STAT3 (Y705).

Treatment of hypoxic (Fig. 4C, 4D) or normoxic hWJMSCs (Fig. 4G, 4H) with $500 \mathrm{ng} / \mathrm{ml} \mathrm{rFGF-17} \mathrm{for} 48 \mathrm{~h}$ resulted in increased expression of phospho-ERK1/2 (Thr202/ Tyr204), whereas phospho-AKT (S473) and phosphoSTAT3 (Y705) were not changed. Based on the relationship between high proliferation and expression of FGF-17 from hypoxic hWJ-MSCs at late passages, we investigated whether FGF-17 was associated with senescence of normoxic and hypoxic hWJ-MSCs. However, expression of senescence-related proteins p21, p27, and p53 was not 
A

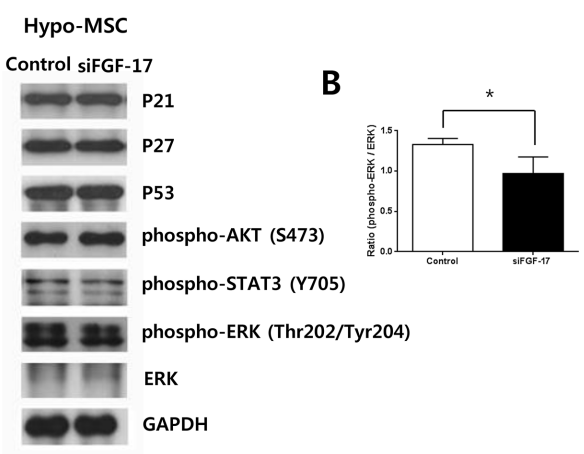

E

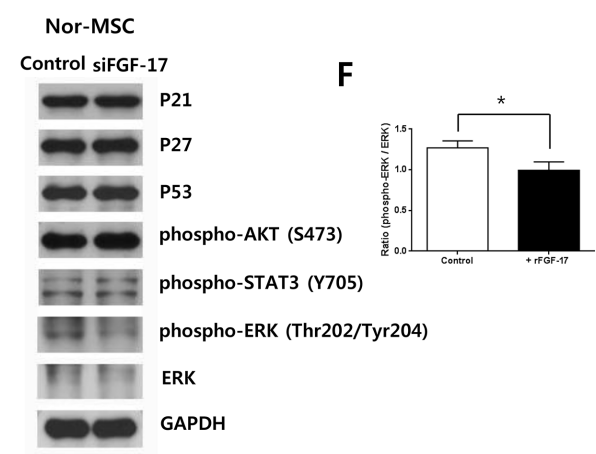

C

Hypo-MSC

Control +rFGF-17

$\longrightarrow$ P21

P27

P53

phospho-AKT (S473)

EZ Phospho-STAT3 (Y705)

D

N1.0.N phospho-ERK (Thr202/Tyr204)

HIIT ERK

GAPDH

G

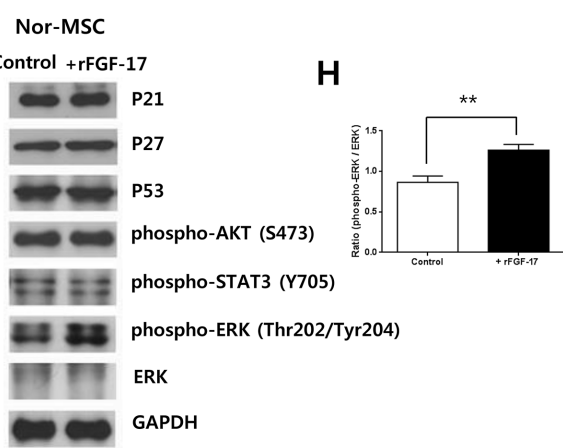

Fig. 4. Effects of FGF-17 on signal transduction in hypoxic and normoxic hWJ-MSCs. (A) Expression of proteins p21, p27, p53, phospho-AKT, phospho-STAT3, phospho-ERK, ERK and GAPDH in hypoxic hWJ-MSCs transfected with siFGF-17. (B) Relative expression of phospho-ERK in hypoxic hWJ-MSCs transfected with siFGF-17 $\left(n=3,{ }^{*} p<0.05\right.$, t-test). Control, hypoxic hWJ-MSC only; siFGF-17, hypoxic hWJ-MSCs with siFGF-17. (C) Expression of proteins p21, p27, p53, phospho-AKT, phospho-STAT3, phospho-ERK, ERK and GAPDH in hypoxic hWJ-MSCs treated with $500 \mathrm{ng} / \mathrm{ml}$ of rFGF-17. (D) Relative expression of phospho-ERK in hypoxic hWJ-MSCs treated with $500 \mathrm{ng} / \mathrm{ml}$ of rFGF-17 ( $n=3, * p<0.05$, t-test). Control, hypoxic hWJ-MSC only; +rFGF-17, hypoxic hWJ-MSCs with rFGF-17. (E) Expression of proteins p21, p27, p53, phospho-AKT, phospho-STAT3, phospho-ERK, ERK and GAPDH in normoxic hWJ-MSCs transfected with siFGF-17. (F) Relative expression of phospho-ERK in normoxic hWJ-MSCs transfected with siFGF-17 ( $n=3,{ }^{*} p<0.05$, t-test). Control, normoxic hWJ-MSC only; siFGF-17, normoxic hWJ-MSCs with siFGF-17. (G) Expression of proteins p21, p27, p53, phospho-AKT, phospho-STAT3, phospho-ERK, ERK and GAPDH in normoxic hWJ-MSCs treated with $500 \mathrm{ng} / \mathrm{ml}$ of rFGF-17. (H) Relative expression of phospho-ERK in normoxic hWJ-MSCs treated with $500 \mathrm{ng} / \mathrm{ml}$ of $\mathrm{rFGF}-17\left(\mathrm{n}=3,{ }^{*} \mathrm{p}<0.01\right.$, t-test). Control, normoxic hWJ-MSC only; +rFGF-17, normoxic hWJ-MSCs with rFGF-17. Nor-MSC, normoxic mesenchymal stem cells; Hypo-MSC, hypoxic mesenchymal stem cells.

changed in hypoxic hWJ-MSCs transfected with siFGF-17 (Fig. 4A) and treated with rFGF-17 (Fig. 4C) or in normoxic hWJ-MSCs treated with siFGF-17 (Fig. 4E) and transfected with rFGF-17 (Fig. 4G).

\section{Effects of FGF-17 on characterization of hWJ-MSCs}

To investigate the effect of FGF-17 on phenotypic characterization of hWJ-MSCs, we analyzed phenotype markers of not only hypoxic hWJ-MSCs transfected with siFGF-17 and treated with rFGF-17 (Fig. 5A, 5B) but also normoxic hWJ-MSCs transfected with siFGF-17 and treated with rFGF-17 (Fig. 5C, 5D). Under both conditions, phenotypes were not changed by FGF-17. The increased expression of osteogenesis-related gene $A L P$, Runx2, and chondrogenesis-related genes Chondroadherin in hypoxic
hWJ-MSCs was significantly decreased by knockdown of FGF-17 (Fig. 5E), and expression of Adiponectin and SOX9 was decreased but without significance (Fig. 5E). When rFGF-17 was treated to hypoxic hWJ-MSCs, Chondroadherin was increased (Fig. 5F). When FGF-17 was knocked down in normoxic hWJ-MSCs, expression of adipogenesis-related gene Adiponectin, osteogenesis-related gene Runx2, and chondrogenesis-related genes Chondroadherin were increased, whereas chondrogenesis-related genes SOX 9 was decreased (Fig. 5G). Expression of the adipogenesis-related gene adiponectin, osteogenesis-related gene Runx2, and chondrogenesis-related genes chondroadherin and SOX9 was significantly decreased in normoxic hWJ-MSCs treated with $\mathrm{rFGF}-17$ and expression of the osteogenesis-related gene $A L P$ was decreased without significance (Fig. $5 \mathrm{H}$ ). 
A

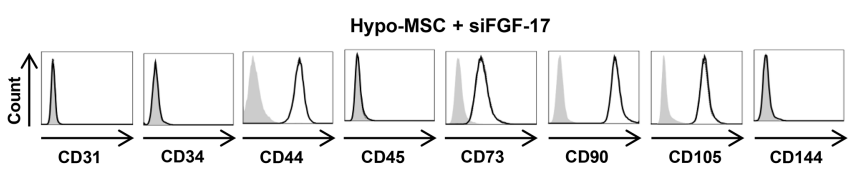

B

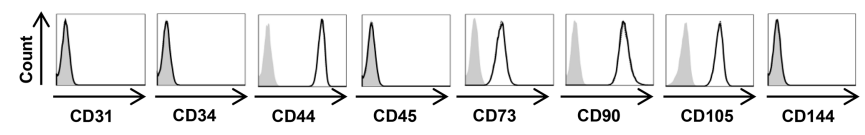

C

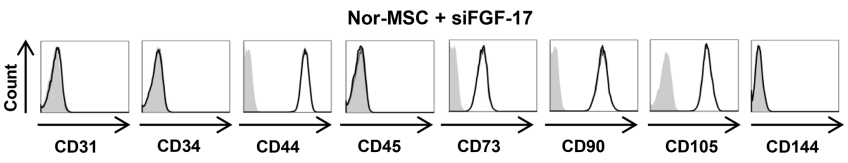

D

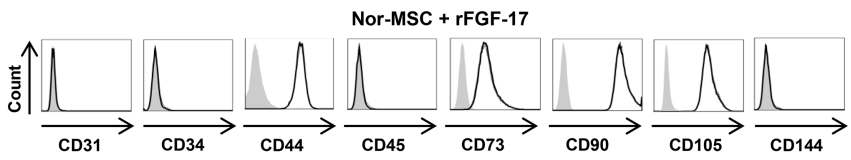

$\mathbf{F}$

E

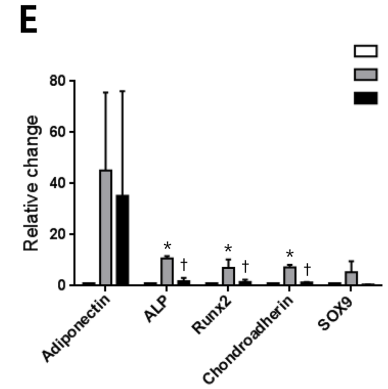

G

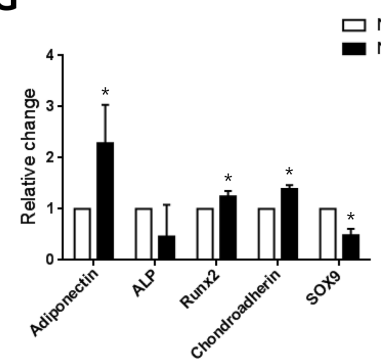

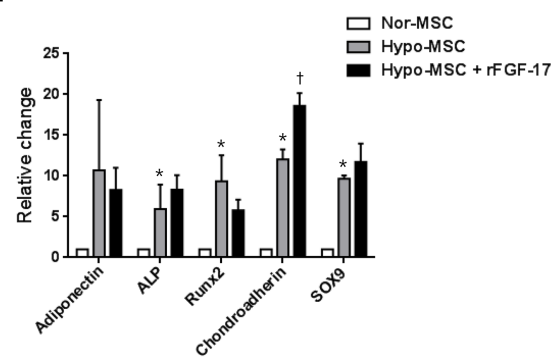

H

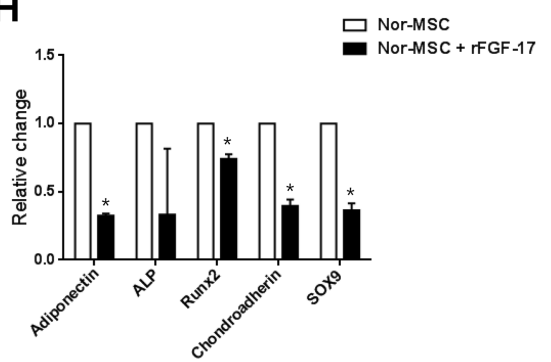

Fig. 5. Effects of FGF-17 on phenotypic characterization of hypoxic and normoxic hWJ-MSCs. (A, B) Negative phenotype markers (CD31, CD34, CD45, and CD144) and positive phenotype markers (CD44, CD73, CD90, and CD105) were analyzed in hypoxic hWJ-MSCs treated with siFGF-17 or rFGF-17 with specific antibody staining and flow cytometry. The gray bar corresponds to the isotype negative control, the dotted line corresponds to hypoxic hWJ-MSCs only, the strong black line indicates hypoxic hWJ-MSCs transfected with siFGF-17 or treated with $500 \mathrm{ng} / \mathrm{ml}$ of rFGF-17. (C, D) The same phenotype markers were analyzed in normoxic hWJ-MSCs treated with siFGF-17 or rFGF-17 with specific antibody staining and flow cytometry. The gray bar corresponds to the isotype negative control, the dotted line corresponds to normoxic hWJ-MSCs only, and the strong black line indicates normoxic hWJ-MSCs transfected with siFGF-17 or treated with $500 \mathrm{ng} / \mathrm{ml}$ of rFGF-17. (E) Adipogenesis-related gene adiponectin, osteogenesis-related genes $A L P$ and Runx2, and chondrogenesis genes chondroadherin and SOX9 were analyzed in hypoxic hWJ-MSCs transfected with siFGF-17 by QRT-PCR assay. Results are expressed as the mean \pm SEM from three independent experiments $\left(n=3,{ }^{*} p<0.05\right.$; Nor-MSC vs. Hypo-MSC, ${ }^{\dagger} p<0.05$; Hypo-MSC vs. Hypo-MSC + siFGF-17, t-test). (F) The same differentiation-related genes were analyzed in hypoxic hWJ-MSCs treated with $500 \mathrm{ng} / \mathrm{ml}$ of $\mathrm{rFGF}-17$. Results are expressed as the mean \pm SEM from three independent experiments $\left(n=3,{ }^{*} p<0.05\right.$; Nor-MSC vs. Hypo-MSC, ${ }^{\dagger} p<0.05$; Hypo-MSC vs. Hypo-MSC+rFGF-17, t-test). (G) The same differentiation-related genes were analyzed in normoxic hWJ-MSCs transfected with siFGF-17 by QRT-PCR assay. Results are expressed as the mean \pm SEM from three independent experiments $\left(n=3,{ }^{*} p<0.05\right.$; Nor-MSC vs. Nor-MSC+ siFGF-17, t-test). (H) The same differentiation-related genes were analyzed in normoxic hWJ-MSCs treated with $500 \mathrm{ng} / \mathrm{ml}$ of $\mathrm{rFGF}-17$. Results are expressed as the mean \pm SEM from three independent experiments $\left(n=3,{ }^{*} p<0.05\right.$; Nor-MSC vs. Nor-MSC+rFGF-17, t-test). Nor-MSC, normoxic mesenchymal stem cells; Hypo-MSC, hypoxic mesenchymal stem cells. 


\section{Discussion}

In this study we show that increased expression of FGF-17 in CM from hypoxic hWJ-MSCs plays a role in maintenance of high proliferation of human mesenchymal stem cells at late passages.

In general, it is well known that the proliferation and survival of cells decreases with increased passage numbers under normal cell culture conditions (18). The mechanisms related to decreased cell proliferation have been reported to be senescence (18), apoptosis (18), DNA mutation (19), and cell transformation (20). In contrast, it has been reported that hypoxic cell culture conditions not only enhance proliferation and survival of cells, but also influence the secretome of mesenchymal stem cells (10-12). According to our protein antibody array, secretory FGF-17 from hypoxic hWJ-MSCs was highly expressed at passage 10 (late passage), and knockdown of FGF-17 decreased cell proliferation of hypoxic hWJ-MSCs. These findings indicate that FGF-17 from hypoxic hWJ-MSCs plays a role in maintaining high proliferation at late passages. In addition, treatment of normoxic hWJ-MSCs at passage 7 (middle passage) with $250 \mathrm{ng} / \mathrm{ml}$ or $500 \mathrm{ng} / \mathrm{ml} \mathrm{rFGF-17} \mathrm{increased}$ cell proliferation, whereas $1,000 \mathrm{ng} / \mathrm{ml}$ rFGF-17 did not further increase proliferation. This may suggest that there is a threshold in the working concentration of FGF-17 for effects on normoxic hWJ-MSCs at the cellular level.

According to protein antibody array, VEGF, IGFBP-3, IL-1 R3, Chordin-Like 1, and EN-RAGE were upregulated at least 2-fold in CM from hypoxic hWJ-MSCs at passage 10 compared with normoxic hWJ-MSCs. It has been reported that VEGF and Chordin-Like 1 are related to increased cell proliferation $(21,22)$ and these may be additional candidate molecules for the maintenance of high proliferation of hypoxic hWJ-MSCs at late passages. Interestingly, previous studies showed that IGFBP-3 and EN-RAGE play a role in inhibiting cell proliferation (23, 24). It is possible that the positive effect of FGF-17, VEGF, and Chordin-Like 1 overcome the negative effect of IGFBP-3 and EN-RAGE for maintenance of high proliferation in late passages of hypoxic hWJ-MSCs. We will further investigate the roles of these molecules.

In a previous study, FGF-4 and associated activation of the ERK1/2 pathway triggered a switch from self-renewal to lineage commitment in embryonic stem cells (25). In contrast to FGF-4, even though treatment with FGF-17 increased the expression of phospho-ERK1/2 in normoxic hWJ-MSCs, the expression of differentiation genes was decreased. This suggests that FGF-4 and FGF-17 may have different roles in stem cell function even though they are both members of the FGF family. It has been reported that expression of FGF-2, FGF-11 and FGF-23 included in fibroblast growth factor family were regulated by hypoxia-inducible factor (HIF-1 $\alpha$ ) in hypoxic condition (26-28). For further study, we will investigate the relation between FGF-17 and HIF-1 $\alpha$.

We presumed that the co-relationship between high expression of FGF-17 and maintenance of cell proliferation at late passages might result from inhibition of cell senescence by FGF-17. However, expression of senescence-related proteins ( $\mathrm{p} 21, \mathrm{p} 27$, and p53) was not changed in normoxic hWJ-MSCs treated with siFGF-17 and rFGF-17 at passage 7 or hypoxic hWJ-MSCs treated with siFGF-17 and $\mathrm{rFGF}-17$ at passage 10.

Furthermore, under the same conditions the phenotypes of normoxic hWJ-MSCs were not altered and expression levels of differentiation-related genes adiponectin (adipogenesis), Runx2 (osteogenesis), and chondroadherin and SOX9 (chondrogenesis) were decreased by treatment of rFGF-17. These results provide supportive evidence for the use of $\mathrm{rFGF}-17$ as a supplement in culture medium of human mesenchymal stem cells in order to enhance the expansion of cells with stemness.

Interestingly, in hypoxic hWJ-MSCs the expression of differentiation genes was increased (significantly for $A L P$, Runx2 and chondroadherin; non-significantly for adiponectin and SOX9) compared with normoxic hWJ-MSCs, whereas knockdown of FGF-17 in hypoxic hWJ-MSCs decreased the expression of these molecules (significantly for $A L P$, Runx2, and chondroadherin; non-significantly for adiponectin and SOX9). This suggests that the effects of FGF-17 on differentiation-related genes are different between normoxic and hypoxic hWJ-MSCs and further studies will be necessary to investigate the mechanisms underlying these differential activities.

In conclusion, FGF-17 from hypoxic hWJ-MSCs plays a role in maintenance of their high proliferation at late passages through the ERK1/2 pathway. And it will be useful as a supplement for the maintenance of proliferation and expansion of hWJ-MSCs under long-term culture conditions.

\section{Acknowledgments}

This research was supported by a grant from the Korea Health Technology R\&D Project through the Korea Health Industry Development Institute (KHIDI), Ministry of Health and Welfare, Republic of Korea (HI17C1728), and by a grant from the Korean Health Technology R\&D Project, Ministry of Health and Welfare, Republic of Korea (HI14C3484). 


\section{Potential Conflicts of Interest}

The authors have no conflicting financial interest.

\section{Supplementary Materials}

Supplementary data including two figures can be found with this article online at http://pdf.medrang.co.kr/paper/ pdf/IJSC/IJSC-12-s18042.pdf.

\section{References}

1. Liu Y, Yan W, Tohme S, Chen M, Fu Y, Tian D, Lotze $M$, Tang D, Tsung A. Hypoxia induced HMGB1 and mitochondrial DNA interactions mediate tumor growth in hepatocellular carcinoma through Toll-like receptor 9. J Hepatol 2015;63:114-121

2. Panigrahi GK, Praharaj PP, Peak TC, Long J, Singh R, Rhim JS, Abd Elmageed ZY, Deep G. Hypoxia-induced exosome secretion promotes survival of African-American and Caucasian prostate cancer cells. Sci Rep 2018;8:3853

3. Porter KM, Kang BY, Adesina SE, Murphy TC, Hart CM, Sutliff RL. Chronic hypoxia promotes pulmonary artery endothelial cell proliferation through $\mathrm{H} 2 \mathrm{O} 2$-induced 5-lipoxygenase. PLoS One 2014;9:e98532

4. Chai X, Sun D, Han Q, Yi L, Wu Y, Liu X. Hypoxia induces pulmonary arterial fibroblast proliferation, migration, differentiation and vascular remodeling via the PI3K/ Akt/p70S6K signaling pathway. Int J Mol Med 2018;41: 2461-2472

5. Chen R, Liu Y, Su Q, Yang Y, Wang L, Ma S, Yan J, Xue F, Wang J. Hypoxia stimulates proliferation of rat neural stem/progenitor cells by regulating mir-21: an in vitro study. Neurosci Lett 2017;661:71-76

6. Nekanti U, Dastidar S, Venugopal P, Totey S, Ta M. Increased proliferation and analysis of differential gene expression in human Wharton's jelly-derived mesenchymal stromal cells under hypoxia. Int J Biol Sci 2010;6:499-512

7. Tsai CC, Chen YJ, Yew TL, Chen LL, Wang JY, Chiu CH, Hung SC. Hypoxia inhibits senescence and maintains mesenchymal stem cell properties through down-regulation of E2A-p21 by HIF-TWIST. Blood 2011;117:459-469

8. Gordan JD, Bertout JA, Hu CJ, Diehl JA, Simon MC. HIF-2alpha promotes hypoxic cell proliferation by enhancing c-myc transcriptional activity. Cancer Cell 2007;11:335-347

9. Zhang H, Nan W, Song X, Wang S, Si H, Li G. Knockdown of HIF-1 $\alpha$ inhibits the proliferation and migration of outer root sheath cells exposed to hypoxia in vitro: an involvement of Shh pathway. Life Sci 2017;191:82-89

10. Bader AM, Klose K, Bieback K, Korinth D, Schneider M, Seifert M, Choi YH, Kurtz A, Falk V, Stamm C. Hypoxic preconditioning increases survival and pro-angiogenic capacity of human cord blood mesenchymal stromal cells in vitro. PLoS One 2015;10:e138477

11. Kim J, Ma T. Autocrine fibroblast growth factor 2-mediated interactions between human mesenchymal stem cells and the extracellular matrix under varying oxygen tension. J Cell Biochem 2013;114:716-727

12. Qin HH, Filippi C, Sun S, Lehec S, Dhawan A, Hughes $\mathrm{RD}$. Hypoxic preconditioning potentiates the trophic effects of mesenchymal stem cells on co-cultured human primary hepatocytes. Stem Cell Res Ther 2015;6:237

13. Hoshikawa M, Ohbayashi N, Yonamine A, Konishi M, Ozaki K, Fukui S, Itoh N. Structure and expression of a novel fibroblast growth factor, FGF-17, preferentially expressed in the embryonic brain. Biochem Biophys Res Commun 1998;244:187-191

14. Granerus M, Engström W. Dual effects of four members of the fibroblast growth factor member family on multiplication and motility in human teratocarcinoma cells in vitro. Anticancer Res 2000;20:3527-3531

15. Nezu M, Tomonaga T, Sakai C, Ishii A, Itoga S, Nishimura M, Matsuo Y, Tagawa M, Nomura F. Expression of the fetal-oncogenic fibroblast growth factor-8/17/18 subfamily in human hematopoietic tumors. Biochem Biophys Res Commun 2005;335:843-849

16. Fortin D, Rom E, Sun H, Yayon A, Bansal R. Distinct fibroblast growth factor (FGF)/FGF receptor signaling pairs initiate diverse cellular responses in the oligodendrocyte lineage. J Neurosci 2005;25:7470-7479

17. Kwon S, Ki SM, Park SE, Kim MJ, Hyung B, Lee NK, Shim S, Choi BO, Na DL, Lee JE, Chang JW. Anti- apoptotic effects of human Wharton's jelly-derived mesenchymal stem cells on skeletal muscle cells mediated via secretion of XCL1. Mol Ther 2016;24:1550-1560

18. Mammone T, Gan D, Foyouzi-Youssefi R. Apoptotic cell death increases with senescence in normal human dermal fibroblast cultures. Cell Biol Int 2006;30:903-909

19. Kim M, Rhee JK, Choi H, Kwon A, Kim J, Lee GD, Jekarl DW, Lee S, Kim Y, Kim TM. Passage-dependent accumulation of somatic mutations in mesenchymal stromal cells during in vitro culture revealed by whole genome sequencing. Sci Rep 2017;7:14508

20. Crowder SW, Horton LW, Lee SH, McClain CM, Hawkins OE, Palmer AM, Bae H, Richmond A, Sung HJ. Passagedependent cancerous transformation of human mesenchymal stem cells under carcinogenic hypoxia. FASEB J 2013;27:2788-2798

21. Fernandes H, Dechering K, van Someren E, Steeghs I, Apotheker M, Mentink A, van Blitterswijk C, de Boer J. Effect of chordin-like 1 on MC3T3-E1 and human mesenchymal stem cells. Cells Tissues Organs 2010;191:443-452

22. Yun SP, Lee MY, Ryu JM, Song CH, Han HJ. Role of HIF-1alpha and VEGF in human mesenchymal stem cell proliferation by 17beta-estradiol: involvement of PKC, PI3K/Akt, and MAPKs. Am J Physiol Cell Physiol 2009; 296:C317-C326

23. Cheng GS, Zhang YS, Zhang TT, He L, Wang XY. Bone marrow-derived mesenchymal stem cells modified with IGFBP-3 inhibit the proliferation of pulmonary artery smooth muscle cells. Int J Mol Med 2017;39:223-230 
24. Hofmann Bowman M, Wilk J, Heydemann A, Kim G, Rehman J, Lodato JA, Raman J, McNally EM. S100A12 mediates aortic wall remodeling and aortic aneurysm. Circ Res 2010;106:145-154

25. Kunath T, Saba-El-Leil MK, Almousailleakh M, Wray J, Meloche S, Smith A. FGF stimulation of the Erk1/2 signalling cascade triggers transition of pluripotent embryonic stem cells from self-renewal to lineage commitment. Development 2007;134:2895-2902

26. Conte C, Riant E, Toutain C, Pujol F, Arnal JF, Lenfant F, Prats AC. FGF2 translationally induced by hypoxia is involved in negative and positive feedback loops with HIF1alpha. PLoS One 2008;3:e3078

27. Yang J, Kim WJ, Jun HO, Lee EJ, Lee KW, Jeong JY, Lee SW. Hypoxia-induced fibroblast growth factor 11 stimulates capillary-like endothelial tube formation. Oncol Rep 2015;34:2745-2751

28. Zhang Q, Doucet M, Tomlinson RE, Han X, Quarles LD, Collins MT, Clemens TL. The hypoxia-inducible factor- $1 \alpha$ activates ectopic production of fibroblast growth factor 23 in tumor-induced osteomalacia. Bone Res 2016;4:16011 